\title{
Developing a Framework for E-Healthcare Applications using the Semantic Internet of Things
}

\author{
Sabah Saad \\ King Abdulaziz University \\ Jeddah, Saudi Arabia
}

\author{
Bassam A. Zafar \\ Faculty of Computing and \\ Information Technology \\ King Abdulaziz University, Jeddah, \\ Saudi Arabia
}

\author{
Ahmed Mueen \\ King Abdulaziz University \\ Jeddah,Saudi Arabia
}

\begin{abstract}
In this paper, discuss the semantic concept, where it found to enrich the logically and conveniently of information accessed capability through web services doing by human or automated using tools. The semantic potentials were based on schemes and formats of data integrated and shared among web, these formats and schemes called ontologies. This technology provides interoperability between medical IoT devices in the healthcare system. The aim of this paper is the effort to developing a framework for e-healthcare applications using the semantic Internet of Things. Extend these ontology descriptions toward the specificity of the healthcare domain. It is to design and implement a framework that holds healthcare data to be then easily used by e-healthcare applications by mapping data into the ontology entities and properties. The objectives of the e-health ontology are to increase access to health care for patients in remote areas, to improve quality of care for patients.
\end{abstract}

\section{General Terms}

Semantic Web

\section{Keywords}

Semantic Web, Ontology, Ontology Languages, XML, RDF, OWL

\section{INTRODUCTION}

The form of using the web today's associated with capture data, search for knowledge, communicate with others, and view and buying products and materials. Thus, the information tools and services will suffer inconsistency, weakly structured documents and inconvenient context as required [1]. The need of machine accessible supportive framework to realized complemented web content emerged, because the existing web structure was meaningless to computers and machines to achieve interoperability of human and machines in a significant way [2].

The researchers and scientist raised and developed techniques to have a formal structure for information available on the web content that machine can process, interpret and connect it, thus called Semantic Web [3]. Semantic web eliminates the correlation structure obstacles in collecting data process from various and diverse sources [4]. The semantic web is constructed through ontologies that support semantic annotation. Ontologies are the agreed structure, concept, relation and attributes for document content in the same domain that allows and enhance shared and reuse of information among the users [3].

The study aims to discover the various languages supported ontology and its data structural concept over the web that benefits semantic. It also aims to satisfy and achieve study objectives, the research will utilize the descriptive methodology using previous literature works and related studies.

The rest of this paper is structured as in following. In section 2 make some literature review about semantic web and ontologies technologies. In section 3, present design of healthcare ontologies and framework architecture that capture the knowledge of medical monitoring devices that will be utilized to produce the e-health system. That will assist patient, also extend SSN ontology to describe medical sensor and IoT devices and the observation and build e-health ontology. In section 4 present the concept of mapping between ontologies by building the relationship between concepts.

\section{LITERATURE REVIEW}

\subsection{Semantic web}

As recently raised the world-wide-web shared data through linked and hypertext process that made it as a big library contains data and documents, where the key activity for business and individual concerns involved in obtaining, evoking and preserving information and knowledge [1]. The focus initiated on the semantic web by Tim Berners - Lee, who state that semantic web which provides machine access information and processing based on machine processable semantic. The growth of integrated efforts that aimed to state semantics for all digital content from various resource and origins, performed and represented in a related matter to fully unleashed the automated services that depend on information and knowledge to raise the interoperability between human and machine [5].

The Web semantic or as called the web of Data is an extended evolution of web that offers exploration, extracting and retrieving a resource from a heterogeneous and distributive resource in view of delivering web supported intelligent semantics extraction enhanced information retrieval, increase precision and raise the level of system interoperability [6]. The vision of Web semantic is pervasive and rapidly deployed in wide application and service, this vision will be a written theory without using ontologies, and ontology is the pillar of Semantic Web vision and application. OWL has significantly featured and extra vocabulary and restriction to provide an explicit description and the relationships. The main advantages of semantic description to supplies a formal promotion for reasoning algorithms.

\subsection{Ontologies}

Ontology known as information and computer science term. Ontology in computer defined as the concept's specification and explicit formal structure [1]. Where the formal referred to machine readable, a concept referred to abstract term for event or phenomena, explicitly referred to expect concept related to the same domain [5]. Respectively, the role of ontology in the 
semantic web is in allowable interoperability through the shared understanding in domains among various distributed and heterogeneous resources, the meaning of semantic agreement in a domain, which is the key feature of semantic web [7]. The ontology used to define commonly shared understanding between communicating parties on purpose to promote the reused and exchange of knowledge and information.

There is no specific, united defined stage or cycles appropriate to employ in all research and application domains. However, there are some steps that cannot be avoided when building an ontology as [8]:

- Seeking for requirements, why do we need the ontology?

- Gathering the knowledge, proper information, concept and terms about a specific domain.

- Design the formal descriptive structure that represents the main concepts and their properties and defines what the ontology languages needed? How do we build the concepts, entities, relation and attributes? What the ontology tools needed?

- Developing the structure, build the relationships and add the detailed to the formal structure.

- Verify the structure to ensure the consistency and checkout any error occurs to be then modified.

- Publishing and implementing the ontology, deployed ontology application services.

Furthermore, W3C classifies OWL to three sub languages OWL Lite which is the simple form of OWL contains class hierarchy and simple constraints, OWL DL used description logic to exploit relation between objects, and OWL Full which considers the highest optimum in expressiveness and syntax [8]. In favorable comparison between previously mentioned ontology languages in term of features, table 1 Show that OWL is the best following to RDF and XML respectively.

Table 1. Comparison Table in Features ontology language provision

\begin{tabular}{|l|l|l|l|}
\hline Features & XML & RDF & OWL \\
\hline Work as Middleware & $\sqrt{ }$ & $\sqrt{2}$ & $\sqrt{ }$ \\
\hline Markup language & $\sqrt{ }$ & $\sqrt{ }$ & $\sqrt{ }$ \\
\hline $\begin{array}{l}\text { Sharing information among } \\
\text { various application }\end{array}$ & $\sqrt{ }$ & $\sqrt{ }$ & $\sqrt{ }$ \\
\hline Based on Data Type & $\sqrt{ }$ & $\times$ & $\times$ \\
\hline Has a limited-on syntax schema & $\sqrt{ }$ & $\times$ & $\times$ \\
\hline Support Semantic & $\times$ & $\sqrt{ }$ & $\sqrt{ }$ \\
\hline $\begin{array}{l}\text { Secure and reliable through } \\
\text { data exchanging among } \\
\text { application }\end{array}$ & $\times$ & $\sqrt{ }$ & $\sqrt{ }$ \\
\hline Processes Meta data & $\times$ & $\sqrt{ }$ & $\sqrt{ }$ \\
\hline Model data & $\times$ & $\sqrt{ }$ & $\sqrt{ }$ \\
\hline Independent in Domains & $\times$ & $\sqrt{ }$ & $\sqrt{ }$ \\
\hline Has rules and grammar bases & $\times$ & $\sqrt{ }$ & $\sqrt{ }$ \\
\hline OIL based (Ontology Inference & $\times$ & $\times$ & $\sqrt{ }$ \\
\hline
\end{tabular}

\begin{tabular}{|l|l|l|l|}
\hline Layer ) & & & \multicolumn{2}{|l|}{} \\
\hline Express Logical Statement & $\times$ & $\times$ & $\sqrt{ }$ \\
\hline Terminology richness & $\times$ & $\times$ & $\sqrt{ }$ \\
\hline
\end{tabular}

Recently, the emerging and developing of sematic web and ontology concepts have a significant impact on knowledge representation particularly when this knowledge used in decision support system. These semantic technologies provide the medical field with significant improvement that helps in arrange and process a large amount of data inaccurately and speedily [1].

The author in [9] discusses semantic technologies in the healthcare field by build knowledge base ontology to smart hospital. They develop ontology as sets of concepts and defined the relation between them. They present ontology as the backbone to the semantic interoperability in the smart hospital the author defines the main benefit of ontology from their point of view to specify a set of concepts to be reused in other application.

The author in [2] builds a drug recommendation system for the diabetic patient. The aim of their paper is to help the clinicians to take a correct decision. This study involves two ontologies patient ontology that contain patients personal information, test and drugs the other ontology is drugs antdiabetic ontology that acts as an exporter of treatment knowledge as glucose level.

McGuinness et al. [10] introduced the web-based browser merging ontology called Chimaera, which developed in the Stanford University Knowledge System Laboratory (KSL), where Chimaera is built in order to work with various ontologies and handles any OKBC compliant representation system. Chimera capable to work with fifteen different input formats in addition to OKBC compliant format, includes but not limited to ANSI KIF, Ontolingua, Protégé, CLASSIC, and so forth.

MOMIS is defined in the scholar work of Beneventano et al. [11], which is notated for Mediator Environment for Multiple Information Sources. MOMIS based on the global virtual view (GVV) of the information source, which is induced regardless of its location, and data heterogeneity. Furthermore, there are some research works utilized the linguistic in benefits of the ontology mapping, which mainly lies on the grammatical forms and context information of the representing language [12]. LILY is one of those ontologies that mainly depends on the linguistics of language [13]. LILY illustrated in the Wang and $\mathrm{Xu}$ [14] works, where the mapping system established based on the linguistic features and the information structure of the ontology which according to both the sub semantic graph is built. LILY utilized numerous techniques through performing mapping to ensure the precise alignment among the concepts and property pairs.

\subsection{Elements of the E-health system}

A traditional E-health system is encompassed by three main elements. This ontology has adopted the tree areas of e-health to provide different applications in the health care sector. Adaptation of the three elements of e-health is aimed at providing efficient and enhanced quality care for patients with chronic illness. It ensures that all elements involved in enhancing the management of a chronic illness are covered for the provision of better health care to these patients. 
Therefore, this ontology has catered for the three elements of e-health for a guaranteed quality care for patients.

The real-time application in e-health can be used for consultation, diagnosis, and treatment of patients in some cases. The store-and-forward mode of the ehealth system is effective in providing reference information regarding a specific condition. Patient data is transmitted to a health care provider remotely for the purposes of tracking the development and management of chronic illness. The telehealth ontology utilizes the availability of the growing mobile health technologies in the market that will be utilized by patients to track their health[14].

\section{HEALTHCARE ONTOLOGY}

Ontology designs form an essential tool in creating a health care system used for patient monitoring and intervention plans [12]. Ontology has an ability to capture a knowledge-based decision support which is simple, accurate, and reliable. The decision support system has a reasoning process that enables the identification of different patient conditions in order to create an intervention plan.

In order to efficiently cater to the dire need of quality care for chronically ill patients, there is a need for a system that will be used to create a personalized knowledge-based decision support for the patients. The system will only highlight the individual disease, symptoms of a patient, and the interventions for the specific patient. The decision support system will be used to critically analyze various recommendations for each patient with adherence to their individual cases [15]. Therefore, this project is focused on developing a health care ontology that is personalized and knowledge-based. Each aspect of the ontology properly evaluates information regarding the patient in order to come up with individual intervention plans. Patient condition is analyzed using all the past and current medical history. By putting into consideration the signs and symptoms that a patient exhibits, it is possible to come up with accurate and up-to-date information regarding their health.

Ontology is basically a health model that is knowledge-based with concepts of a given clinical condition which has a relation with other related clinical cases [12]. Decision support systems are essential in helping health care professionals to make relevant decisions regarding a particular disease or syndrome [16].

A knowledge-based system is created that will provide necessary medical support with a knowledge structure that will help medical practitioners in making decisions regarding recommended therapeutic measures, diagnosis, and correct interventions. The system provides a centered support for patients with chronic illness by personalizing the signs and symptoms of the particular patient[17].

This paper has utilized ontology design to produce an e-health system that will assist patients with chronic diseases. An e- health system is based on main information regarding a patient or a condition that is available in the main health care system. Patients can be able to receive quality medical care from their homes without any difficulties. Local health centers will also be able to coordinate with main medical hospitals to provide medical assistance to patients without a need for referrals. The e-health system utilizes all phases of ontology design to come up with an accurate and realistic system with relevant information concerning a particular condition. Therefore, personalization became an essential element in making the ontology functional in an efficient, accurate, and reliable manner. Each entity in personalization is based on accurate information describing either a particular case scenario or intervention methods[17].

The objectives of the telehealth ontology are to increase access to health care for patients in remote areas, to improve quality care for patients with chronic diseases, to provide home-based care for patients with chronic illness, to facilitate monitoring of patients with chronic illness, to reduce the probability of readmission of patients with chronic illnesses, to ease congestion in hospitals, to increase patient satisfaction.

The ontology design of this project is developed using protégé 5.2 under the URL: www.semanticweb.org/Hospital\#. Development of the ontology is mainly focused on providing formal intervention plans for health care specifically focused on patients with chronic illness.

Representatives give relevant information concerning respective illnesses, syndrome, and social challenges of patients [18]. An effective telehealth system has the following main entities and classes as highlighted below:

- Chronic diseases such as; diabetes (type $1 \& 2$ ), hypertension, cardiovascular disease, heart failure, chronic obstructive pulmonary disease, different types of dementia, depression, and cognitive impairment.

- Chronic syndromes that affect patients with chronic diseases which include; immobility and cognitive impairment which are the most common issues that affect patients with chronic diseases.

- Patients are also affected socially with issues like mental illness, lack of family support, insufficient funds, and unconducive environment.

The ontology design has relationships between different classes and their subclasses as shown in fig. 1 below.

It is notable that for the ontology to be accurate and reliable, there is a need to critically analyze all clinical information regarding a certain disease. The relationship between different entries is crucial towards building a functional e-health system. The telehealth system will act as a link between the patient and health care professionals who are responsible for giving recommendations regarding a patient's progress. 


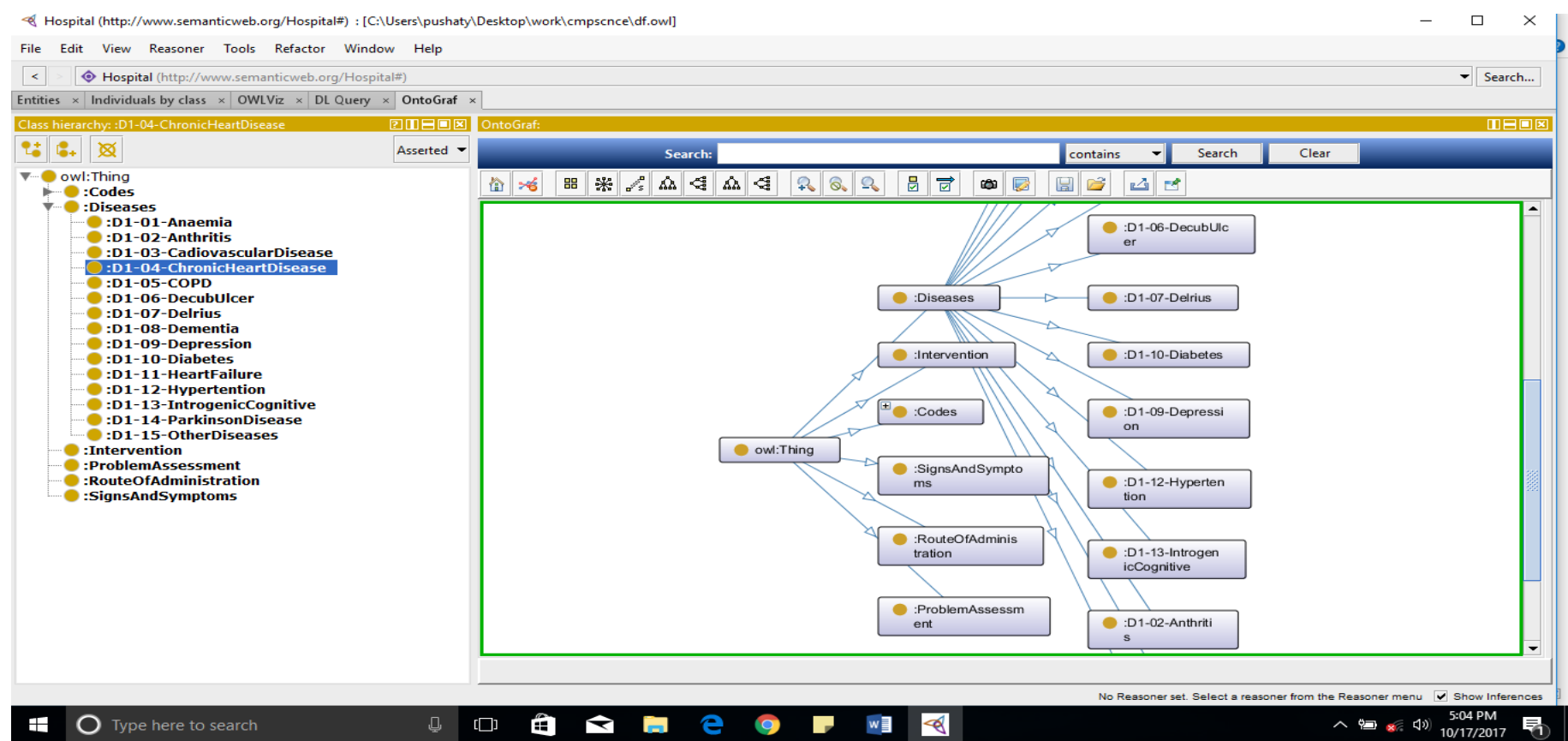

Fig 1: Relations between the ontology concepts

Fig. 2 shows a description of a patient's interaction with health care providers through the telehealth ontology. The telehealth system has an interface for the patient and the health providers where a patient's personal medical records are stored in a personalized data server that is updated with a patient's medical progress. Regular updates are sent to the telehealth system from patient data in order to determine relevant medical progress[19].

Basically, the patient enters his or her information in the patient interface. Daily progress of the patient is captured through IOT monitoring clinical devices or equipment, the data is then sent to the main server. The main server is based on the hospital system which contains relevant information concerning the patient's medical history, details of diseases and their symptoms, personalized intervention plans, and personalized quality care. Depending on the data received from a patient, the analysis is done in the main server to determine the progress of a patient and current clinical development. A doctor is able to view the results of a patient and give formal clinical recommendations which are sent to the patient. Therefore, the telehealth system simplifies the management of a chronic disease. The information extracted relating to diabetes' signs and symptoms allow health care providers to reach conclusions regarding a patient's condition through rdfs property in the ontology.

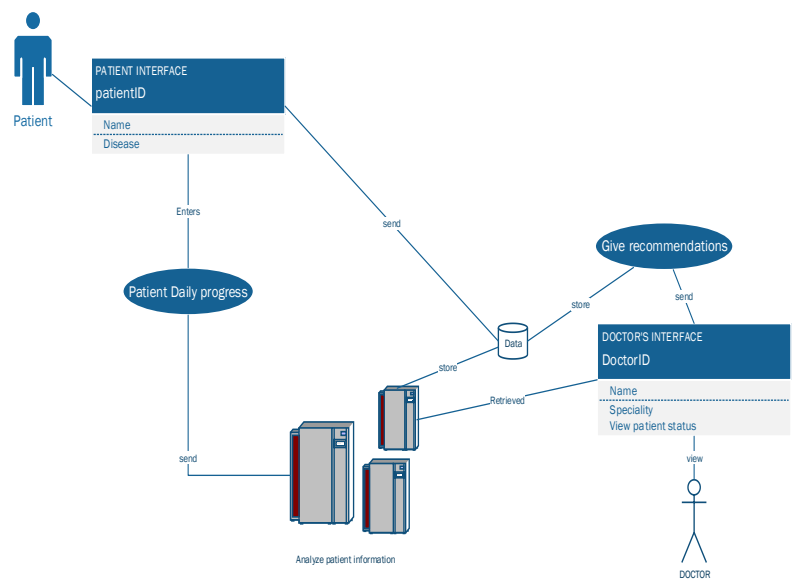

Fig 2: E-health data flow between patient and doctor

In this paper develop framework architecture to deal with IOT and semantic ontology bout E-health care sector to provide the interoperability. The main features are related to extensibility and interoperability so that it can essay integrate different vendors devices, new IoT devices, protocols and new healthcare applications. The collected data from IoT devices especially related to personal information and vital sign organized in SSN ontology as Feature of interest (Featureofinterest) class The object whose attribute is being evaluated or examined as an Observation to reach the Result, Observable attribute (Observableproperty) class: which defined as" An observable Feature, attributes of a FeatureOfInterest thing, Observation class defined as " Act of carrying out an (Observation) and mapping by building a relationship between similar and connected concepts With Ehealth ontology. E-health ontology only concerned on the following classes:

Person Class embraces the persons, who were motoring, IoT_Device Class this class represented the all IoT device, Vital_Sign Class: is a class where the vital sign of patient was recorded. this framework provides the interoperability by applying export and import query with the medical system. 


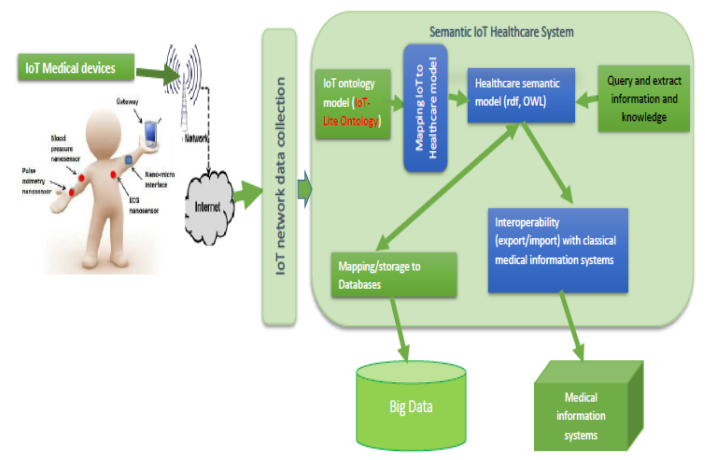

Fig 3: IoT Healthcare System architecture based on ontology modeling

\subsection{Mapping between the ontology}

The w3c semantic sensor network incubator group assign semantic sensor network (SSN) ontology to represent sensors ability, their attributes and their result observation. The group agreements were to construct ontology to represent sensors. They use the expansive qualifier of the sensor's concepts and sub-concepts assignee later for more explanation. In the SNN ontology, the main concepts and their relation were defined like sensors, properties' result of sensors as observation and the sensing system. They defined measuring, operating, existence condition and deployment. Finally, implementing stimulus-sensor-observation ontology design pattern [20].

SSN is defined as main concepts into 41 concepts and 39 properties it is constructed by ontology design pattern (ODP) characterize the relation of stimulus, sensors and observation design pattern (SSO) as a common base for SNN ontology [20]. Stimuli that events present any alteration on the environmental situation (SSN:Stimulus) the stimulus consider as proxy (SSN:isproxyfor) observation properties (SSN:Property). Sensors (SSN:Sensors) present the objects that sense (SSN:detects) (SSN:Sensing) observation output and convert it other representation (SSN:SensorOutput). Observation that connects the events of stimulus sensors (SSN:observed by) a process (SSN:SensingMethodUsed) output (SSN:observationResult), feature (SSN:featureOfInterest) and attributes of observation (SSN:observationproperty) the observation describe stimuli by sensors as events [15]. The execution and accuracy of sensors influenced by the common environmental situation that know in SSN ontology as sensors measurements capabilities [14]. The ontology specify (SSN:Accuracy , ssn:Drift, ssn:DetectionLimit, ssn:Frequency, ssn:MeasurementRange, ssn:Resolution, ssn:Latency,ssn:ResponseTime, ssn:Precision, ssn:Selectivity and ssn:Sensitivity as sensor measurement capabilities(ssn:measurementcapabilities, ssn:measurmentsproperty) [21].

The observation perspective is the context of explaining stimuli.The ontology model context as (ssn:featureofinterest) observing sensor (ssn:observedBy), property (ssn:observedProperty), method (ssn:sensingMethodUsed) result (ssn:observationResult), quality of the observation (ssn:qualityOfObservation), a time at which the sampling take (ssn:observationSamplingTime) and result became available (ssn:observationResultTime). In SSN, system perspective that builds about the system (ssn:system) to describe sensing infrastructure. System modeled as (ssn:hassubsystem)that have (ssn:has operatingRange)and(ssn:hassurvivalRange)and may be deployed(ssn:hasDeployment). The operating range (ssn:operatinRange)representing a feature of the environmental and the situation in which the system is operating. The survival Range presents the environmental situation to the sensor confronts without any hurt. Deployment perspective (ssn:deployment) is the operation that includes all the stage in the deployed system [21].

Recently, ontology considers as crucial to achieved interoperability across systems specially the heterogeneous systems and the semantic web application [22; 23]. However, the ontology defined as a method that state a clear and distinct description in order to create a common conceptualization shared among various systems [20].

Ontology was spread on various business and application fields, where one model could have multiple ontologies, in such case to perform a task, these ontologies have to support each other and fully understand each, but in real case that one system could embrace various heterogeneous and distributed ontologies which cannot fully support and communicate with each other to support task operating [21], therefore scholars adapts the ontology mapping techniques in order to solve this issue, and guarantees interoperability of systems [22].

\subsubsection{E-health ontology structure}

The ontology mainly defined around more than ten classes such as codes, RouteOfAdminstration, Vital_Sign, Person, Intervention, Iot _devices and etc. However, these classes have subclasses, for example, the iot_Devicess class has three subclasses, including the following: Wearables, Medical _devices and internal_Embedded_Medical_Devices. In the context of this ontology, the research only concerned on the following classes:

Person Class: this class embraces the persons, who were motoring in the system. In the current ontology, there are three persons were monitoring. However, these persons are defined as a member of class or individual of class - the ground level of the ontology.

Iot_Device Class: including subclasses or modules, this class represented the all IoT device currently in use, including the internal embedded medical devices, medical devices and wearable devices. However, the medical devices module have only one individual "Device2" in the current case, as well as the "Wearables" class which has only one individual "device1". In contrary, the system does not include any embedded devices, based on the fact that the class of the internal_Embedded_medical device does not have any individuals.

Vital_Sign Class: is a class where the vital sign of patient was recorded, the vital sign has five classes: Glucose level_in_blood; blood_pressure, Pulse_rate and so forth. Only consider the pulse rate sign which includes four individuals, representing four readings from Pulse reading medical device in different type, the data property defined as an integer. 


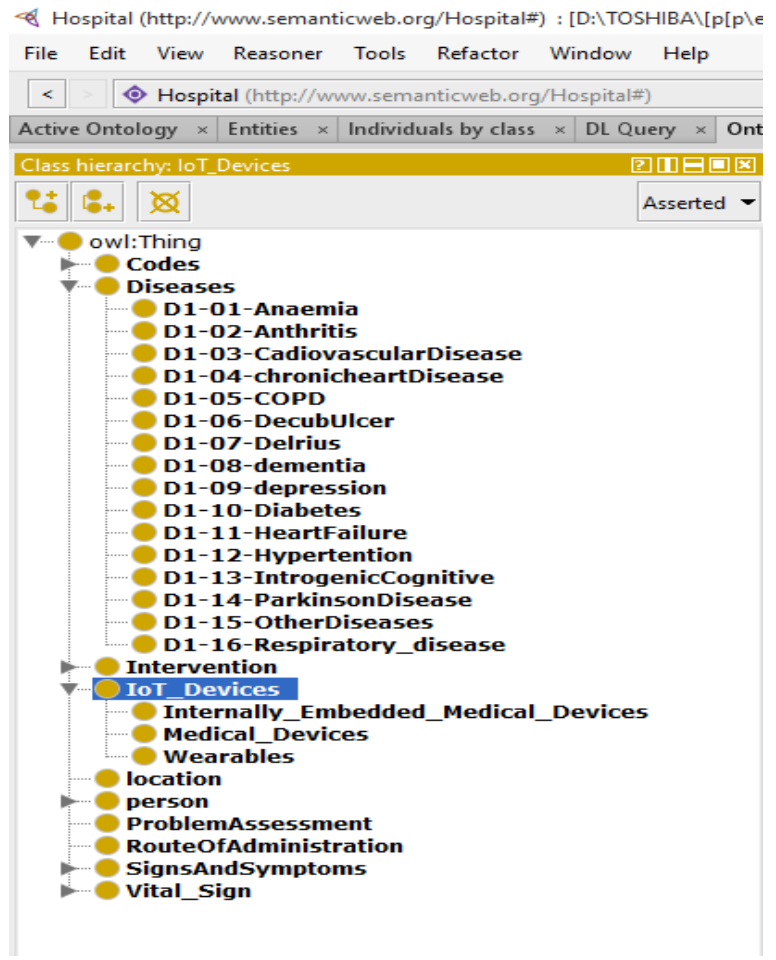

Fig 4: structure of E-health ontology

\subsubsection{SSN Ontology structure}

SSN ontology used here is the SoSa/SSN ontology, which introduced as combined of two common ontologies; the W3C (World Wide Web Consortium) and OGC (Open Geospatial Consortium) Spatial Data on the Web (SDW) Working Group. The combination ontology used only to describes sensors, actuators, samplers and its observation, actuation and sampling activities. This ontology is qualified to support an enormous range of application and observation drove ontology engineering, which explained it's appropriate with health -care motoring application field. SSN ontology classes are mentioned in:

Feature of interest (Featureofinterest) class: this class the object whose attribute is being evaluated or examined as an Observation to reach the Result or whose attribute is being addressed by a sensor. For example: "when calculating the rise of a tree, the high is the ObservableProperty, $40 \mathrm{~m}$ as an outcome of the Observation, and the tree as objects of the FeatureOfInterest ". Which in our case has two properties as individuals Person\#1, Person\#2.

Observable property (Observableproperty) class: which defined as" An observable Feature, attributes of a FeatureOfInterest thing, as in previous example The high of a tree is a sample of observable properties, like in our ontology Pulse_Rate\#1, Pulse_Rate\#2.

Observation class: realized as "work of execution to evaluate the value of a feature of a FeatureOfInterest object. connect to a Sensor to characterize what the Observation produced and how; connect to an ObservableProperty to characterize what the result is an evaluated of, in this case it includes three medical observations. as an example " Medical_observation\#1000, Medical_observation\#1001, Medical_observation\#1002".

The sensor: is an entity platform that hosts other sub entities such as healthcare devices, in current cases, it includes three health care devices.

\section{MANUAL MAPPING}

We make a manual mapping by building a relationship between similar and connected concepts. This implementation is divided into different mapping steps based on which segment the mapping occurs. It includes prefix mapping, base mapping, properties mapping, classes mapping, and individual mapping.

Prefix mapping: the transformation of prefix from SSN ontology to E-health ontology. For example, the prefix of SSN ontology @prefix : <http://www.coode.org/ontologies\#> is transformed to @prefix : <http://www.semanticweb.org/Hospital\#> in E-health ontology.

Base mapping: the transformation of base prefix from SSN ontology to E-health ontology. For example, the base prefix of SSN ontology@base <http://www.co-ode.org/ontologies\#> is transformed to @ base <http://www.semanticweb.org/Hospital\#> in E-health ontology.

Annotation properties mapping: the transformation of properties from SSN ontology to E-health ontology. For example, SSN ontology includes properties like hasProperty, but in E-health ontology, the properties are more specified such as Has_location.. The properties in SSN ontology are called annotation properties, while they are divided into object properties and data properties in E-health ontology.

Object properties: the transformation of object properties from SSN HealthCare to E-health ontology. Some properties are combined into one property, whereas others may further divided.

Data properties: the transformation of data properties from SSN HealthCare to E-health ontology. Similar to object properties, the properties are further divided.

Classes mapping: the transformation of classes from SSN HealthCare to E-health ontology. A class person is the same in both ontologies but in E-healthcare ontology it is specified as a patient. Pulse rate class in SSN ontology is divided into multiple individuals in E-health ontology including vital sign, blood pressure, and body temperature, the glucose level in blood, pulse rate, and respiration rate. Similarly, medical observations are converted into signs and symptoms but with more specified types like diabetes, heart disease, and hypertension. Healthcare devices are divided into wearables and internally embedded medical devices. It can be concluded that many classes are defined in E-health ontology that was not defined in SSN ontology. These classes include H:ProblemAssessment, H:RouteOfAdministration, H:location, H:patient, and many others.

Individual mapping: Consequently, the mapping of individuals from SSN HealthCare to E-health ontology. Devices are created by both ontologies, but the location of devices is defined as a class in E-health ontology. Persons are also created by both ontologies.

Ontology mapping and integration: To fill data of E-health ontology from SSN ontology, need a separate language to provide data translation from IoT devices and individuals of ontology to the medical ontology. Further, data in medical ontology need to be retrieved and queried.

E-health ontology contains heterogeneous sensors from different industrial production data with multiple data formats. Thus, it is much needed to have device interoperability and 
data normalization based on manual interpretation or explicit programming. The implementation of data transmission from one ontology to another is not an easy task so it requires providing semantic interoperability between different ontologies. In smart healthcare ontologies, need a semantic interoperability platform based on semantic web technologies and using standardized healthcare information exchange.

In ontology mapping and integration, the data is represented, managed and queried. So it includes ontology mapping and query. The shared vocabulary and concepts help to understand the properties and relations among ontologies. Semantic interoperability can be achieved through different query languages such as SPARQL. This language is one of the query languages developed for RDF files. To resolve the issue of ontology translation between different knowledge domains, SPARQL stream can query continuous data [24].

The data can be extract from SSN based ontology (SSN healthcare-case study) and use SPARQL for data extraction with a select statement. Likewise, the statement can be insert to add individuals from SSN ontology to Medical global healthcare ontology. Table 9 shows, in general, the mapping between SSN ontology with E-health ontology including classes, properties, and individuals.

Table 2: the mapping between SSN ontology with E-health ontology including classes, properties, and individuals

\begin{tabular}{|c|c|}
\hline $\begin{array}{l}\text { E-HEALTH } \\
\text { ONTOLOGY }\end{array}$ & SSN ONTOLOGY \\
\hline H: person2 & sosa:Person 2 \\
\hline $\begin{array}{l}\text { H: sign1 } H: \\
\text { pulse rate }\end{array}$ & $\begin{array}{c}\text { sosa:Observation } \\
\text { Observation } 01000\end{array}$ \\
\hline H:sign_value & sosa:hasResult \\
\hline H: signs_from & sosa:madeBySensor \\
\hline H:date & sosa:resultTime \\
\hline H:Devices 1 & Healthcare_devices1 \\
\hline H:Devices 2 & Healthcare_devices 2 \\
\hline H:location1 & $\begin{array}{l}\text { geo:alt } 12.5 \\
\text { geo:lat } 35.8 \\
\text { geo:long }-120.61\end{array}$ \\
\hline H:location2 & $\begin{array}{l}\text { geo:alt } 13 \\
\text { geo:lat } 12.6 \\
\text { geo:long } 35.2\end{array}$ \\
\hline H:latitude & geo:lat \\
\hline H:longitude & geo:long \\
\hline H: pulse rate & sosa:observedProperty \\
\hline H: person & sosa:hasFeatureOfInterest \\
\hline H: person & sosa:Person \\
\hline H:Devices & sosa:madeBySensor \\
\hline
\end{tabular}

\section{E-HEALTH SYSTEM IMPLEMENTATION}

Fig 5 shown the care provider must login to the e-health system by entering the right user name and the password after that the access will be provided to the care provider.

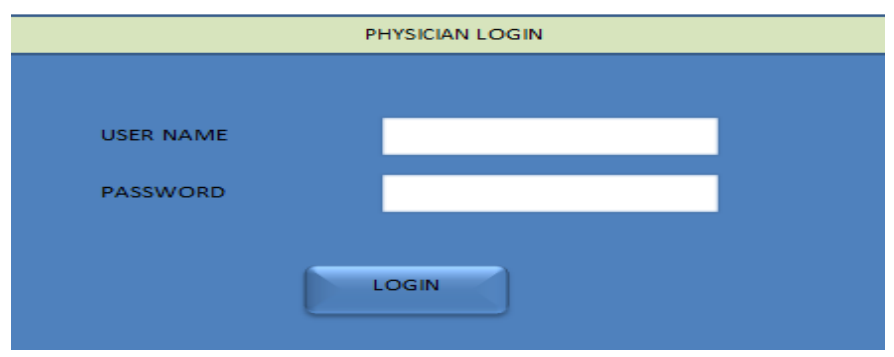

Fig 5: physician login interfaces

When the patient new to the e-health system must register the personal information .this information will be saved in the ontology to be then retrieved by the care provider. This patient's personal information used to complete the medical records when the patient register.

The start diagnosis interfaces allow the physician to start the diagnosis operation by when the physician select the disease option some of the chronic diseases will provide as shown in Fig 6. The value of vital sign of monitoring the patient operation will return to the physician. Then, a list of signs and symptoms will appear to be selected depending on the patient situation and the physician observation.

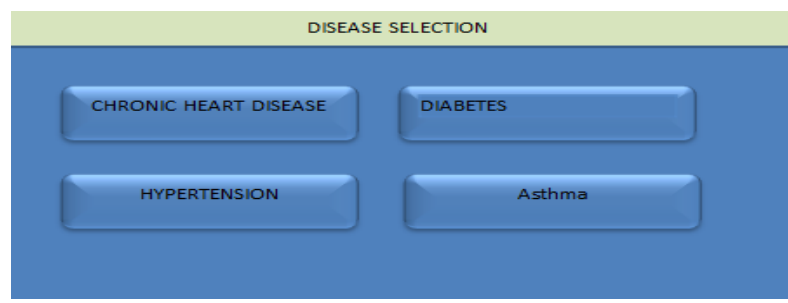

Fig 6: the disease selection interfaces

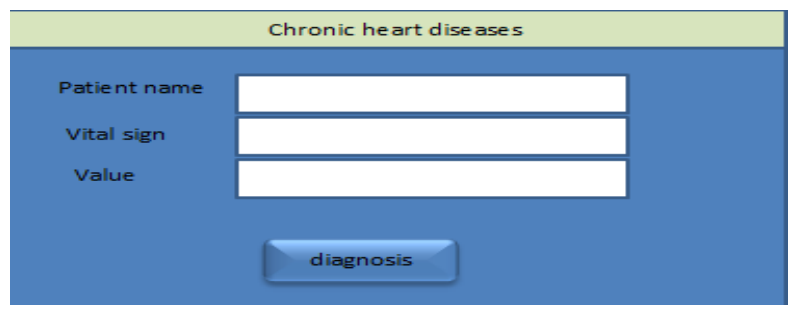

Fig 7: Chronic heart diseases interface

In the framework that was developed to improve the e-health system particularly in monitoring chronic disease patient by provide permanent vital signs monitoring and the observation of patient situation Through the use of IoT medical devices and represent this data in semantic Ontology to retrieve process when needed by a physician as in Fig 7. The final diagnosis of the patient's situation monitoring of the vital signs and disease if there is some recommendation and alert message to the patient represent in this interface. The diagnosis operation will depend on vital sign value and signs and symptoms associated with it. 

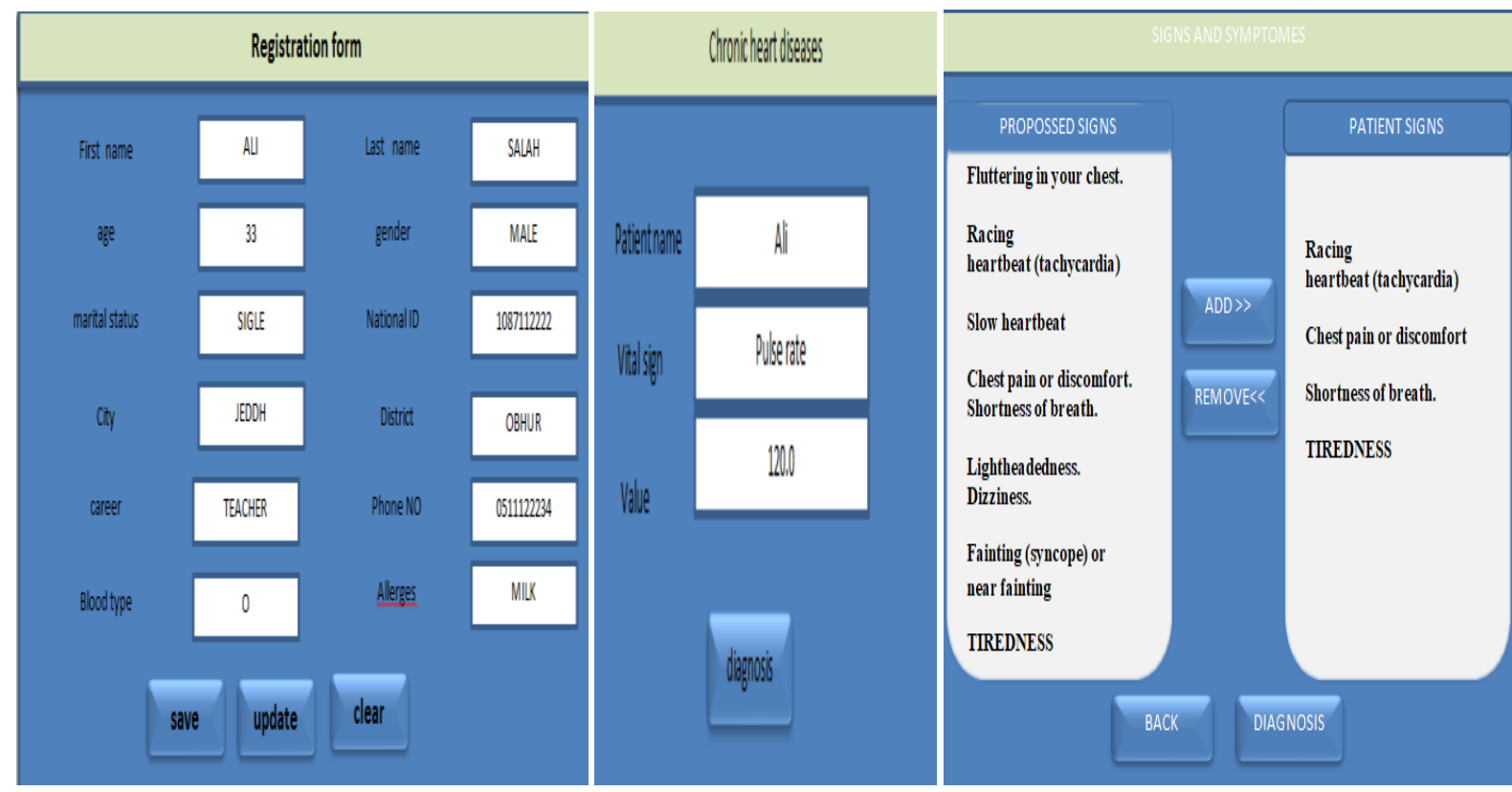

\begin{tabular}{|c|c|}
\hline \multicolumn{2}{|l|}{ Ontology } \\
\hline 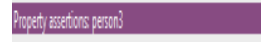 & 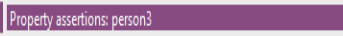 \\
\hline 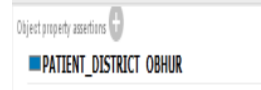 & $\begin{array}{l}\text { object ropenety assetions } 4 \\
\text { EPATIENT_DISTRICT OBHUR } \\
\text { HEART DISEASE SIGNS }\end{array}$ \\
\hline DHAS_CTTY JEDDAH & $\begin{array}{l}\text { SHORTNESS_OF_BREATH } \\
\text { SHESTE }\end{array}$ \\
\hline WHAS_AllerGGS MILK & EHEART_DISEASE_SIGNS RAPID_HEARTBEAT \\
\hline BLOOD_TYPE 0 & =HEART_DISEASE_SIGWS CHEST_PAIN \\
\hline IPATIENICARRER TECACER & HAS_CITY JEDDAH \\
\hline mGet_vital_signs signt & $\begin{array}{l}\text { DHEART_DISEASE_SIGWS IIREDNESS } \\
\text { DBLOOD_TYPE } 0\end{array}$ \\
\hline Pat putat asting 0 & EPATIENI_CARRER TEACHER \\
\hline 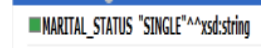 & EGet_vital_signs sign 4 \\
\hline Gender "Male" rxscisting & Data prppeti assations $\rightarrow$ \\
\hline WATIONAL_ID 1087112222 & mARITAL_STATUS "SINGLE"^^xSd:String \\
\hline IPHONE_NO 0511112234 & Gender "MALE"^^xsd:String \\
\hline Wname 'Ali" $\wedge$ xsodsting & -NATONAL_ID 10871112222 \\
\hline Iage $33^{\prime \prime} \wedge x x d i n t$ & DPHONE_NO 0511122234 \\
\hline 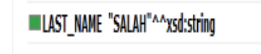 & Dage "33"^^xst:int \\
\hline & - LAST_NAME "SALAH"^^xsd:String \\
\hline
\end{tabular}
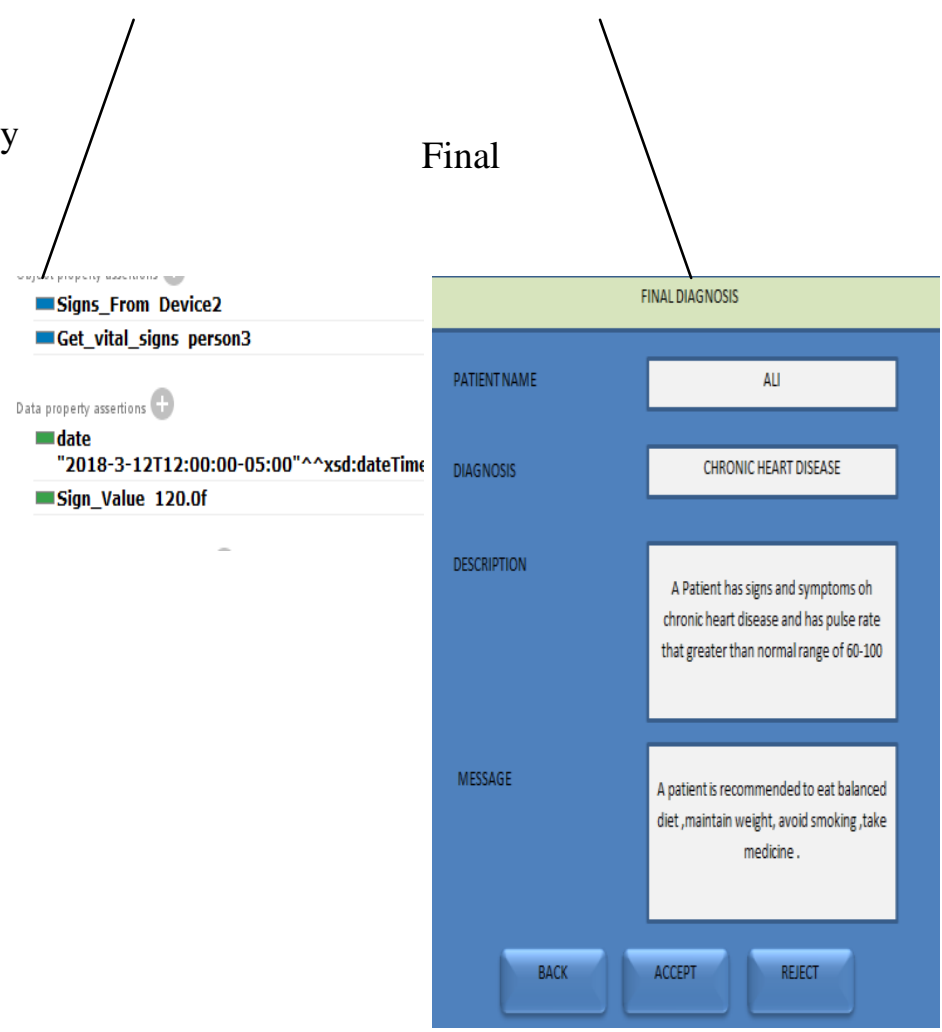

\section{Figure 8: Formalization of Patient Data and Retrieve Interface}

Here we have case study as the patient is 'Ali' from Jeddah with pulse rate value 120 pulse per second that measure remotely by using IoT devices. The physician observation was this patient have chronic heart disease with signs and symptoms as Racing heartbeat, Chest pain or discomfort, Shortness of breath, tiredness.so the e-health system will give recommendation message to the patient as described in Fig 8 that describes the whole system

\section{CONCLUSION}

In this paper, focusing on developing a framework to assist in improving the health care sector depend on IOT technologies as sensors to collect patient's data and provide remote and continuous monitoring of patient status. Also, applied semantic web technologies to provide the interoperability between a Heterogeneity cases in IOT sources of data. SSN Sensors utilized in many applications like medical care, security and metrology and an environmental supervision. Semantic technologies act as mean to provide 
interoperability for the sensing system. Semantic technologies help in integrating, processing and managing sensors and their output result. This permits the user to avoid technical issues and focusing on the concepts.

One ontology may be not enough to provide the support for some application so multiplies ontology need to access. therefore adapt the ontology mapping techniques in order to solve this issue, and guarantees interoperability. Make a manual mapping by building a relationship between similar and connected concepts. This implementation is divided into different mapping steps based on which segment the mapping occurs. It includes prefix mapping, base mapping, properties mapping, classes mapping, and individual mapping.

\section{ACKNOWLEDGMENTS}

Our thanks to the experts who have contributed towards this development who provided insight and expertise that greatly assisted the research.

\section{REFERENCES}

[1] Antonio, G. Harmelen, F. (2008). A Semantic Web Primer, $2^{\text {nd }}$ Ed, London: The MIT Press.

[2] Bonteceva, K. Cunningham, H. (2003). The Semantic Web: A new Opportunity and Challenge for Human Language Technology, in: Workshop on Human Language Technology for the semantic web and web services second international semantic web conference ISWC03, Sanibel Island, Florida

[3] Amardeilh, F. (2009). Semantic Annotation and Ontology Population In Semantic web engineering in the Knowledge Society, (pp.135 -160). Pennsylvania: IGI Global.

[4] Anderson, J. Rainie,* Lee (2010). The fate of The Sematic Web, Washington DC: Pew Research Center internet \&American Life Project

[5] Ding, Y. Fensel, D. Stork, H. (2003). The Semantic Web: From Concept to precept. OGAI, 22(1-2).

[6] Macgregor, G.(2009). E-source Management and the Semantic Web: application of RDF for e-Source discovery. In E-resources Management Handbook (pp.120). Electronic pubkisher: UKSG.

[7] Taye, M. (2010). Understanding Semantic Wen and ontologies: Theory and application. Journal of computing, vol. 2, No.2.

[8] Gómez-Pérez, A., Fernández-López, M. and Corcho, O. (2010). Ontological engineering. 1st ed. London: Springer

[9] Yadagiri, N. Ramesh, P. (2013).Semantic Web and the Libraries: An Overview, international Journal of library Science, 7(1).

[10] McGuinness, R. Fikes, J. Rice and S. Wilder, "The Chimaera Ontology Environment", in HE SEVENTEENTH NATIONAL CONFERENCE ON ARTIFICIAL INTELLIGENCE-AAAI-00, Austin Texas, 2000, pp. 1-2.
[11] Beneventano, M. Orsini, L. Po, A. Sala and S. Sorrentino, "An Ontology-Based Data Integration System for Data and Multimedia Sources", in IEEE International Conference on Semantic Computing ICSC2009, Berkeley, California, 2009.

[12] a. J. M. C. Rosse, "“A reference ontology for biomedical informatics"," The Foundational Model of Anatomy. J Biomed Inform, no. 36, p. 478-500, 2003.

[13] Bikaks, N. Tsinaraki, C. Gioldasis, N. Stavrakantonakis, I. Christodoulakis, S. (2013). The XML and Semantic Web World: Technologies, interoperability and integration a survey of state of the Art. In: Semantic Hyper/Multi-media Adaptation: Schemes and Applications, electronic publisher: Springer.

[14] M. C. W. E. J Kvedar, "Connected health: a review of technologies and strategies to improve patient care with telemedicine and telehealth". Health Affairs., 2014.

[15] A. Jovic, M. Prcela and D. Gamberger, "Ontologies in Medical Knowledge Representation", in Information Technology Interfaces, 2007. ITI 2007. 29th International Conference on, Cavtat, Croatia, 2007.

[16] S. Anand and A. Verma, "Development of Ontology for Smart Hospital and Implementation using UML and RDF", IJCSI International Journal of Computer Science Issues, vol. 7, no. 5, 2010.

[17] H. L. a. R. Glueckauf, "Research on telehealth and chronic medical conditions: Critical review, key issues, and future directions". Rehabilitation., 2002.

[18] P. Wang and B. Xu, "Lily: ontology alignment results for OAEI 2009", in the 4th International Workshop on Ontology Matching (OM-2009), Chantilly, USA, 2009.

[19] M. C. W. E. J Kvedar, "Connected health: a review of technologies and strategies to improve patient care with telemedicine and telehealth". Health Affairs., 2014

[20] K. Cahill, "Patient Observation (Vital Signs) Policy Adult", Sydney South West Area Health Service, 2010.

[21] A. Goldfain, B. Smith, S. Arabandi, M. Brochhausen and W. R. Hogan, "Vital Sign Ontology", Proceedings of the Workshop on Bio-Ontologies, ISMB, 2011.

[22] A. M. J. D. P. \&. H. A. Doan, "Learning to map between ontologies on the semantic web," in In Proceedings of the 11th international conference on World Wide Web (pp. 662-673), ACM., 2002.

[23] N. S. I. Y. \&. H. H. Choi, "A survey on ontology mapping," ACM Sigmod Record, vol. 35, no. 3, pp. 3441, 2006.

[24] M. \&. S. Y. Ehrig, "Ontology mapping-an integrated approach," in In European Semantic Web Symposium(pp. 76-91), Springer, Berlin, Heidelberg., 2004. 